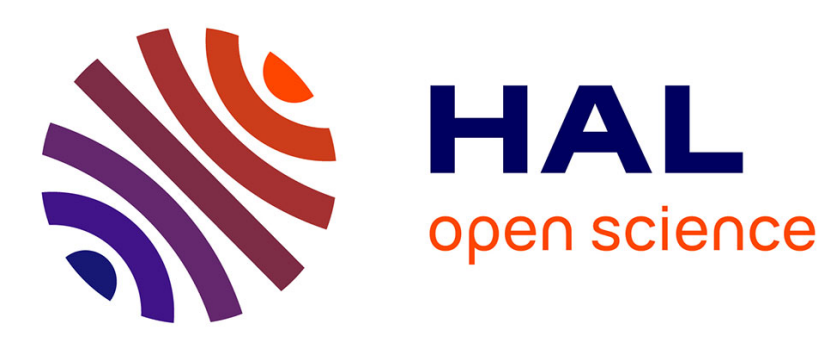

\title{
On Acceleration of a Class of Asymptotically Stable Homogeneous Systems
}

\author{
Denis Efimov, Arie Levant, Andrey Polyakov, Wilfrid Perruquetti
}

\section{To cite this version:}

Denis Efimov, Arie Levant, Andrey Polyakov, Wilfrid Perruquetti. On Acceleration of a Class of Asymptotically Stable Homogeneous Systems. Proc. 55th IEEE Conference on Decision and Control (CDC), Dec 2016, Las Vegas, United States. hal-01371274

\section{HAL Id: hal-01371274 \\ https://hal.inria.fr/hal-01371274}

Submitted on 25 Sep 2016

HAL is a multi-disciplinary open access archive for the deposit and dissemination of scientific research documents, whether they are published or not. The documents may come from teaching and research institutions in France or abroad, or from public or private research centers.
L'archive ouverte pluridisciplinaire HAL, est destinée au dépôt et à la diffusion de documents scientifiques de niveau recherche, publiés ou non, émanant des établissements d'enseignement et de recherche français ou étrangers, des laboratoires publics ou privés. 


\title{
On Acceleration of a Class of Asymptotically Stable Homogeneous Systems
}

\author{
Efimov D., Levant A., Polyakov A., Perruquetti W.
}

\begin{abstract}
A switched supervisory algorithm is proposed, which ensures fixed-time convergence by commutation of finitetime or exponentially stable homogeneous systems of a special class, and a finite-time convergence to the origin by orchestrating among asymptotically stable systems. A particular attention is paid to the case of exponentially stable systems. Performance of the proposed control algorithms is illustrated by computer experiments.
\end{abstract}

\section{INTRODUCTION}

State feedback stabilization of linear or nonlinear systems is a central problem in the control systems theory. There are many methods [1], [6], [8], which are differing by the requirements imposed on the plant model and on the guaranteed performance of the closed-loop system. By performance we understand: robustness with respect to external disturbances, measurement noises and small delays, rate and domain of convergence, overshooting in the system, etc.

The present work studies the problem of adjustment of the rate of convergence in a class of such closed-loop systems, when they are homogeneous [15], [1]. It is a well-known fact that if a system is homogeneous with a negative/zero/positive degree and asymptotically stable, then actually it has a finitetime/exponential/asymptotic rate of convergence (in the case of positive degree the time of convergence to a sphere is uniformly bounded by a constant for any initial conditions, if the system is also locally finite-time converging, then it is called fixed-time stable) [10], [11], [12]. Despite of that, an additional problem of acceleration can be posed [4], [5], i.e. the problem of changing of type of convergence (from exponential to finite-time or fixed-time, for example) by adjustment of parameters.

In [4], [5] for homogeneous systems with a negative degree it has been proposed an algorithm of on-line switching between parameters, which ensures a desired accelerated rate

All authors are with Non-A team @ Inria, Parc Scientifique de la Haute Borne, 40 avenue Halley, 59650 Villeneuve d'Ascq, France and CRIStAL (UMR-CNRS 9189), Ecole Centrale de Lille, Cité Scientifique, 59651 Villeneuve-d'Ascq, France.

D. Efimov and A. Polyakov are with Department of Control Systems and Informatics, University ITMO, 49 av. Kronverkskiy, 197101 Saint Petersburg, Russia.

A. Levant is with School of Mathematical Sciences,Tel-Aviv University, TelAviv 6997801, Israel.

This work was partially supported by ANR 15 CE23 0007 (Project Finite4SoS), the Government of Russian Federation (Grant 074-U01) and the Ministry of Education and Science of Russian Federation (Project 14.Z50.31.0031). of convergence for the closed-loop system. The proposed strategy consists in a proper decreasing the acceleration rate by parameter commutation till the normal one close to the origin. In the present work the following extensions are proposed. First, the algorithms of fixed-time convergence are developed for exponentially converging systems with zero degree (linear systems). Second, the scenario with growing acceleration is also analyzed (in such a case the time when a control with augmented amplitude is applied can be seriously limited). Third, the algorithm of parameter switching for finite-time convergence to the origin is developed for homogeneous systems with zero or positive degree.

The outline of this paper is as follows. Notation and preliminary results are introduced in sections II and III. The precise problem statement is given in Section IV. The proposed supervisory algorithms of switching among different sets of parameters ensuring a required acceleration are presented in Section V. All algorithms are illustrated by computer experiments with a benchmark example. Concluding remarks and discussion appear in Section VI.

\section{NOTATION}

Through the paper the following notation is used:

- $\mathbb{R}_{+}=\{x \in \mathbb{R}: x \geq 0\}$, where $\mathbb{R}$ is the set of real number.

- $|\cdot|$ denotes the absolute value in $\mathbb{R},\|\cdot\|$ denotes the Euclidean norm on $\mathbb{R}^{n}$.

- A continuous function $\alpha: \mathbb{R}_{+} \rightarrow \mathbb{R}_{+}$belongs to the class $\mathcal{K}$ if $\alpha(0)=0$ and the function is strictly increasing. The function $\alpha: \mathbb{R}_{+} \rightarrow \mathbb{R}_{+}$belongs to the class $\mathcal{K}_{\infty}$ if $\alpha \in \mathcal{K}$ and it is increasing to infinity.

- A series of integers $1,2, \ldots, n$ is denoted by $\overline{1, n}$.

\section{PRELIMINARIES}

In this work the following nonlinear system is considered:

$$
\dot{x}(t)=f(x(t)), t \geq 0,
$$

where $x(t) \in \mathbb{R}^{n}$ is the state, $f: \mathbb{R}^{n} \rightarrow \mathbb{R}^{n}$ ensures forward existence of the system solutions at least locally, $f(0)=0$. For an initial condition $x_{0} \in \mathbb{R}^{n}$ define the corresponding solution by $X\left(t, x_{0}\right)$ for any $t \geq 0$ for which the solution exists. If $f$ is discontinuous, then the solutions are understood in the Filippov's sense [7].

Following [14], [8], [12], let $\Omega$ be an open neighborhood of the origin in $\mathbb{R}^{n}$. 
Definition 1. At the steady state $x=0$ the system (1) is said to be

(a) Lyapunov stable if for any $x_{0} \in \Omega$ the solution $X\left(t, x_{0}\right)$ is defined for all $t \geq 0$, and for any $\epsilon>0$ there is $\delta>0$ such that for any $x_{0} \in \Omega$, if $\left\|x_{0}\right\| \leq \delta$ then $\left\|X\left(t, x_{0}\right)\right\| \leq \epsilon$ for all $t \geq 0$

(b) asymptotically stable if it is Lyapunov stable and for any $\kappa>0$ and $\epsilon>0$ there exists $T(\kappa, \epsilon) \geq 0$ such that for any $x_{0} \in \Omega$, if $\left\|x_{0}\right\| \leq \kappa$ then $\left\|X\left(t, x_{0}\right)\right\| \leq \epsilon$ for all $t \geq T(\kappa, \epsilon)$

(c) finite-time stable if it is Lyapunov stable and finitetime converging from $\Omega$, i.e. for any $x_{0} \in \Omega$ there exists $0 \leq T<+\infty$ such that $X\left(t, x_{0}\right)=0$ for all $t \geq T$. The function $T_{0}\left(x_{0}\right)=\inf \left\{T \geq 0: X\left(t, x_{0}\right)=0 \forall t \geq T\right\}$ is called the settling time of the system (1);

(d) fixed-time stable if it is finite-time stable and $\sup _{x_{0} \in \Omega} T_{0}\left(x_{0}\right)<+\infty$.

The set $\Omega$ is called the domain of stability/attraction.

If $\Omega=\mathbb{R}^{n}$, then the corresponding properties are called global Lyapunov stability/asymptotic stability/finitetime/fixed-time stability of (1) at $x=0$.

Similarly stability notions can be defined with respect to a set, by replacing the distance to the origin in Definition 1 with the distance to an invariant set.

\section{A. Weighted homogeneity}

Following [15], [1], [9], for strictly positive numbers $r_{i}$, $i=\overline{1, n}$ called weights and $\lambda>0$, one can define:

- the vector of weights $\mathbf{r}=\left(r_{1}, \ldots, r_{n}\right)^{T}, r_{\max }=$ $\max _{1 \leq j \leq n} r_{j}$ and $r_{\min }=\min _{1 \leq j \leq n} r_{j}$;

- the dilation matrix function $\Lambda_{r}(\lambda)=\operatorname{diag}\left\{\lambda^{r_{i}}\right\}_{i=1}^{n}$, note that $\forall x \in \mathbb{R}^{n}$ and $\forall \lambda>0$ we have $\Lambda_{r}(\lambda) x=$ $\left(\lambda^{r_{1}} x_{1}, \ldots, \lambda^{r_{i}} x_{i}, \ldots, \lambda^{r_{n}} x_{n}\right)^{T}$;

- the $\mathbf{r}$-homogeneous norm $\|x\|_{r}=\left(\sum_{i=1}^{n}\left|x_{i}\right|^{\frac{\rho}{r_{i}}}\right)^{\frac{1}{\rho}}$ for any $x \in \mathbb{R}^{n}$ and $\rho \geq r_{\max }$, then there exist $\underline{\sigma}, \bar{\sigma} \in \mathcal{K}_{\infty}$ such that

$$
\underline{\sigma}\left(\|x\|_{r}\right) \leq\|x\| \leq \bar{\sigma}\left(\|x\|_{r}\right) \quad \forall x \in \mathbb{R}^{n} ;
$$

- the unit sphere and the unit ball in the homogeneous norm $S_{r}=\left\{x \in \mathbb{R}^{n}:\|x\|_{r}=1\right\}$ and $B_{r}(\rho)=\{x \in$ $\left.\mathbb{R}^{n}:\|x\|_{r} \leq \rho\right\}$ for $\rho \geq 0$.

Definition 2. A function $g: \mathbb{R}^{n} \rightarrow \mathbb{R}$ is $\mathbf{r}$-homogeneous with degree $\mu \in \mathbb{R}$ if $\forall x \in \mathbb{R}^{n}$ and $\forall \lambda>0$ we have:

$$
\lambda^{-\mu} g\left(\Lambda_{r}(\lambda) x\right)=g(x) .
$$

A vector field $f: \mathbb{R}^{n} \rightarrow \mathbb{R}^{n}$ is $\mathbf{r}$-homogeneous with degree $\nu \in \mathbb{R}$, with $\nu \geq-r_{\min }$ if $\forall x \in \mathbb{R}^{n}$ and $\forall \lambda>0$ we have:

$$
\lambda^{-\nu} \Lambda_{r}^{-1}(\lambda) f\left(\Lambda_{r}(\lambda) x\right)=f(x),
$$

which is equivalent for $i$-th component of $f$ being a $\mathbf{r}$-homogeneous function of degree $r_{i}+\nu$.

The system (1) is $\mathbf{r}$-homogeneous of degree $\nu$ if the vector field $f$ is $\mathbf{r}$-homogeneous of degree $\nu$.
Theorem 1. [15], [13] For the system (1) with $\mathbf{r}$-homogeneous and continuous function $f$ the following properties are equivalent:

- the system (1) is (locally) asymptotically stable;

- there exists a continuously differentiable $\mathbf{r}-$ homogeneous Lyapunov function $V: \mathbb{R}^{n} \rightarrow \mathbb{R}_{+}$such that

$$
\begin{aligned}
& \alpha_{1}(\|x\|) \leq V(x) \leq \alpha_{2}(\|x\|), L_{f} V(x) \leq-\alpha(\|x\|), \\
& \lambda^{-\mu} V\left(\Lambda_{r}(\lambda) x\right)=V(x), \mu>r_{\max }, \\
& \forall x \in \mathbb{R}^{n} \text { and } \forall \lambda>0, \text { for some } \alpha_{1}, \alpha_{2} \in \mathcal{K}_{\infty} \text { and } \\
& \alpha \in \mathcal{K} \text {. }
\end{aligned}
$$

The requirement on continuity of the function $f$ has been relaxed in [2] (the function $V$ can also be selected smooth).

For $\mathbf{r}$-homogeneous system (1) with degree $\nu$, the solutions also admit a kind of homogeneity as functions of time $\forall x_{0} \in \mathbb{R}^{n}$ and $\forall t \in \mathbb{R}$ :

$$
X\left(t, \Lambda_{r}(\lambda) x_{0}\right)=\Lambda_{r}(\lambda) X\left(\lambda^{\nu} t, x_{0}\right) \quad \forall \lambda>0 .
$$

For $q>1$ define $T_{q}: \mathbb{R}^{n} \rightarrow \mathbb{R}_{+}$such that

$$
\left\|X\left(T_{q}\left(x_{0}\right), x_{0}\right)\right\|_{r}=q^{-1}\left\|x_{0}\right\|_{r},
$$

i.e. it is the function of convergence in $q$ times, then from the properties stated above it is easy to show that it is $\mathbf{r}$-homogeneous with degree $-\nu$ :

$$
T_{q}\left(\Lambda_{r}(\lambda) x_{0}\right)=\lambda^{-\nu} T_{q}\left(x_{0}\right) \quad \forall x_{0} \in \mathbb{R}^{n} \forall \lambda>0 .
$$

This fact follows by applying dilation to the definition expression:

$$
\begin{gathered}
\left\|X\left(T_{q}\left(\Lambda_{r}(\lambda) x_{0}\right), \Lambda_{r}(\lambda) x_{0}\right)\right\|_{r}=q^{-1}\left\|\Lambda_{r}(\lambda) x_{0}\right\|_{r} \\
\left\|X\left(\lambda^{\nu} T_{q}\left(\Lambda_{r}(\lambda) x_{0}\right), x_{0}\right)\right\|_{r}=q^{-1}\left\|x_{0}\right\|_{r} \\
\lambda^{\nu} T_{q}\left(\Lambda_{r}(\lambda) x_{0}\right)=T_{q}\left(x_{0}\right) .
\end{gathered}
$$

\section{PRoblem Statement}

Consider a chain of integrators stabilized in finite time or exponentially by a state feedback $k: \mathbb{R}^{n} \rightarrow \mathbb{R}$ :

$$
\dot{x}(t)=f(x(t))=A x(t)+b k(x(t)), t \geq 0,
$$

where $x(t) \in \mathbb{R}^{n}$ is the state vector, the matrices

$$
A=\left[\begin{array}{cccc}
0 & 1 & 0 \ldots 0 & 0 \\
0 & 0 & 1 \ldots 0 & 0 \\
\vdots & \vdots & \ddots & \vdots \\
0 & 0 & 0 \ldots 0 & 1 \\
0 & 0 & 0 \ldots 0 & 0
\end{array}\right], b=\left[\begin{array}{c}
0 \\
\vdots \\
0 \\
1
\end{array}\right]
$$

are in canonical forms; for $r_{i}=1+(i-1) \nu$ with $i=\overline{1, n}$ and $\nu \in\left[-n^{-1}, 0\right]$ the function $k$ is $\mathbf{r}$-homogeneous of degree $1+n \nu$, i.e.

$$
k\left(\Lambda_{r}(\lambda) x\right)=\lambda^{1+n \nu} k(x) \quad \forall x \in \mathbb{R}^{n} \forall \lambda>0,
$$

then the system (2) is $\mathbf{r}$-homogeneous of degree $\nu$ :

$$
f\left(\Lambda_{r}(\lambda) x\right)=\lambda^{\nu} \Lambda_{r}(\lambda) f(x) \quad \forall x \in \mathbb{R}^{n} \forall \lambda>0 .
$$


Since $\nu \in\left[-n^{-1}, 0\right]$, if (2) is asymptotically stable, then it has either finite-time or exponential rate of convergence [1].

The problem studied in this note: is it possible to ensure a fixed-time stabilization in (2) by switching among different sets of coefficients in the feedback $k$. Such a problem has been already investigated in [4], [5], and following these works instead of (2) we will consider for all integer $i \geq 0$ the system:

$$
\begin{gathered}
\dot{z}(t)=A z(t)+b u\left(\mu_{i}, z(t)\right), t \in\left[t_{i}, t_{i+1}\right), \\
u\left(\mu_{i}, z\right)=\mu_{i}^{n} k\left(M_{i} z\right),
\end{gathered}
$$

where $z(t) \in \mathbb{R}^{n}$ is the state vector, $A, b$ and $k$ are as before; $M_{i}=\operatorname{diag}\left\{\mu_{i}^{1-k}\right\}_{k=1}^{n}$ and $\mu_{i} \geq 1$ is a sequence of parameters, which stay constant on the interval $\left[t_{i}, t_{i+1}\right)$ and change their values at instants $t_{i}, i \geq 0\left(t_{0}=0\right)$. It is required to determine the instants $t_{i}, i \geq 0$ and the discrete-time update law for $\mu_{i}$ such that (3) becomes fixed-time stable. Obviously, (3) is $\mathbf{r}$-homogeneous of degree $\nu$ for any $i \geq 0$.

\section{SUPERVISORY ALGORITHM DESIGN}

Let us introduce a dynamical system

$$
\dot{x}(t)=\mu_{i} f(x(t))=\mu_{i}[A x(t)+b k(x(t))], t \in\left[t_{i}, t_{i+1}\right)
$$

where $\mu_{i}$ is the same as in (3), and after update of $\mu_{i}$ to $\mu_{i+1}$ at the instant of time $t_{i+1}$ we have a state resetting for $x(t)$ :

$$
x\left(t_{i+1}\right)=M_{i+1} M_{i}^{-1} x\left(t_{i+1}^{-}\right),
$$

where $x\left(t_{i+1}^{-}\right)$denotes the right limit of $x(t)$ as $t$ is approaching $t_{i+1}$ from the left. As we can conclude, (4), (5) is a hybrid system, which has to be augmented by rules for assignment of switching instants $t_{i}$ and for update of $\mu_{i}$, for all $i \geq 0$.

Obviously, $z(t)=M_{i}^{-1} x(t)$ for $t \in\left[t_{i}, t_{i+1}\right)$ is the corresponding solution of (3). Therefore, in order to design the supervisory algorithms for selection of $t_{i}$ and $\mu_{i}$ we will consider the hybrid system (4), (5) below.

\section{A. Fixed-time stabilization for $\nu<0$}

In this work the following algorithm is proposed for all $i \geq 0$ :

$$
t_{i+1}=\min _{t \geq t_{i}:\|x(t)\|_{r} \leq q^{-1-i}\|z(0)\|_{r}} t,
$$

where $q>1$ is a tuning parameter, define $i^{*}=$ $\operatorname{ceil}\left[\max \left\{0, \ln _{q}\|z(0)\|_{r}\right\}\right]$ (the function ceil $[s]$ provides the smallest integer bigger than $s$ ) and

$$
\mu_{i}=\left\{\begin{array}{ll}
q^{-i(\alpha-\nu)}\|z(0)\|_{r}^{-\nu} & \text { if } i<i^{*} \\
1 & \text { otherwise }
\end{array},\right.
$$

where $\alpha \in(\nu, 0)$ is another tuning parameter. It is necessary to show that the solutions of (4), (5) are well defined for the supervisory algorithm (6), (7), and that the closed loop system converges to the origin in a fixed time.

Note that for each fixed $\mu_{i}$ the system (4) is converging to the origin (indeed, $X_{\mu_{i}}\left(t, x_{0}\right)=X\left(\mu_{i} t, x_{0}\right)$ where $X_{\mu_{i}}\left(t, x_{0}\right)$ is a solution of (4) and $X\left(t, x_{0}\right)$ is a solution of
(2)), then always there exists an instant $t_{i+1}$ defined by (6) for all $i \geq 0$. Since $\alpha-\nu>0$, from (7) we obtain that $\mu_{i}>1$ for all $i \in\left[0, i^{*}\right)$ and $\mu_{i}=1$ for all $i \geq i^{*}$. Therefore, for all $t \geq t_{i^{*}}$ the system (4), (5), (6), (7) is reduced to (2), which is finite-time stable, and $\left\|x\left(t_{i^{*}}\right)\right\|_{r} \leq 1$. The solutions of this system are well defined for all $t \geq t_{i^{*}}$ (there is no more jump in the state), and it is necessary to consider that happens for $t \in\left[0, t_{i^{*}}\right)$ and estimate the time of convergence to the unit ball $t_{i^{*}}$. To this end, from (7) we obtain for $i \in\left[0, i^{*}-1\right]$ :

$$
\begin{gathered}
M_{i+1} M_{i}^{-1}=\operatorname{diag}\left\{\left(\frac{\mu_{i+1}}{\mu_{i}}\right)^{1-k}\right\}_{k=1}^{n} \\
=\operatorname{diag}\left\{q^{(\alpha-\nu)(k-1)}\right\}_{k=1}^{n},
\end{gathered}
$$

and $M_{i+1} M_{i}^{-1}$ is stretching since $q>1$ and $\alpha-\nu>0$, i.e.

$$
\begin{aligned}
\left\|x\left(t_{i+1}\right)\right\|_{r} & <\left\|x\left(t_{i+1}^{+}\right)\right\|_{r} \leq q^{\frac{(\alpha-\nu)(n-1)}{1+\nu(n-1)}}\left\|x\left(t_{i+1}\right)\right\|_{r} \\
& \leq q^{\frac{(\alpha-\nu)(n-1)}{1+\nu(n-1)}-1-i}\|z(0)\|_{r},
\end{aligned}
$$

where the last step is obtained from (6). This property also implies that $t_{i+1}-t_{i}>0$ and the solutions of the system (4), (5), (6), (7) are well defined.

The instant $t_{i+2}$ will be generated by (6) when $\left\|x\left(t_{i+2}\right)\right\|_{r}=q^{-2-i}\|z(0)\|_{r}$ and on the interval of time $\left[t_{i+1}, t_{i+2}\right)$ the dynamics of $x(t)$ is governed by (4) for $\mu_{i+1}=q^{-(i+1)(\alpha-\nu)}\|z(0)\|_{r}^{-\nu}$. On this interval $\left[t_{i+1}, t_{i+2}\right)$ the state $x(t)$ has to decrease in $\rho=q^{\frac{1+\alpha(n-1)}{1+\nu(n-1)}}$ times, i.e. the norm $\|x(t)\|_{r}$ goes down from the level $q^{\frac{(\alpha-\nu)(n-1)}{1+\nu(n-1)}-1-i}\|z(0)\|_{r}$ till $q^{-2-i}\|z(0)\|_{r}$. Let $T_{\rho}: \mathbb{R}^{n} \rightarrow$ $\mathbb{R}_{+}$be the function of convergence in $\rho>1$ times for (2), then

$$
\begin{aligned}
t_{i+2}-t_{i+1} & =\mu_{i+1}^{-1} T_{\rho}\left(x\left(t_{i+1}\right)\right) \\
& \leq \mu_{i+1}^{-1} q^{\left(1+i-\frac{(\alpha-\nu)(n-1)}{1+\nu(n-1)}\right) \nu}\|z(0)\|_{r}^{-\nu} T_{\rho}(\xi) \\
& \leq q^{\alpha(i+1)-\frac{(\alpha-\nu)(n-1)}{1+\nu(n-1)} \nu} T_{\rho}(\xi)
\end{aligned}
$$

where $z(0)=\Lambda_{r}(\lambda) \xi$ for some $\xi$ from the unit sphere $S_{r}=$ $\left\{\xi \in \mathbb{R}^{n}:\|\xi\|_{r}=1\right\}$. Let $\bar{T}_{\rho}=\sup _{\|\xi\|_{r}=1} T_{\rho}(\xi)$, then

$$
\begin{aligned}
t_{i^{*}} & =\sum_{i=0}^{i^{*}-1}\left(t_{i+1}-t_{i}\right) \\
& \leq \sum_{i=0}^{i^{*}-1} q^{\alpha i-\frac{(\alpha-\nu)(n-1)}{1+\nu(n-1)} \nu} \bar{T}_{\rho} \\
& =q^{-\frac{(\alpha-\nu)(n-1)}{1+\nu(n-1)} \nu} \bar{T}_{\rho} \sum_{i=0}^{i^{*}-1} q^{\alpha i} \\
& \leq \frac{q^{-\frac{(\alpha-\nu)(n-1)}{1+\nu(n-1)} \nu}}{1-q^{\alpha}} \bar{T}_{\rho},
\end{aligned}
$$

where the fact that $q^{\alpha}<1$ has been used on the last step. Therefore, the time of convergence to the unit ball $t_{i^{*}}$ in the system (4), (5), (6), (7) is finite and independent in initial conditions, as it was necessary to show. The following result has been proven (see also [4], [5]). 
Theorem 2. Let for the system (3) with $\nu<0$ the supervisory algorithm be selected as in (6), (7) with $q>1$ and $\nu<$ $\alpha<0$, then the solutions of the closed-loop system are well defined for all $t \geq 0$ and it is fixed-time stable with the settling time

$$
\mathrm{T}^{1}+\frac{q^{-\frac{(\alpha-\nu)(n-1)}{1+\nu(n-1)} \nu}}{1-q^{\alpha}} \bar{T}_{\rho},
$$

where $\mathrm{T}^{1}$ is the time of convergence to the origin from the homogeneous sphere for the system (2) and $\bar{T}_{\rho}=$ $\sup _{\|\xi\|_{r}=1} T_{\rho}(\xi)$ with $\rho=q^{\frac{1+\alpha(n-1)}{1+\nu(n-1)}}$.

Remark 1. The same result can be obtained substituting (7) with

$$
\mu_{i}= \begin{cases}q^{i(\alpha-\nu)}\|z(0)\|_{r}^{-\nu} & \text { if } i<i^{*} \\ 1 & \text { otherwise }\end{cases}
$$

for $\alpha \in(2 \nu, \nu)$.

Remark 2. Clearly,

$$
\mathrm{T}^{1}+\frac{q^{-\frac{(\alpha-\nu)(n-1)}{1+\nu(n-1)} \nu}}{1-q^{\alpha}} \bar{T}_{\rho} \leq\left(1+\frac{q^{-\frac{(\alpha-\nu)(n-1)}{1+\nu(n-1)} \nu}}{1-q^{\alpha}}\right) \mathrm{T}^{1},
$$

and a straightforward analysis shows that the function

$$
\sigma(q)=1+\frac{q^{-\frac{(\alpha-\nu)(n-1)}{1+\nu(n-1)} \nu}}{1-q^{\alpha}}
$$

has its minimum for

$$
q^{*}=\sqrt[-\alpha]{1+\frac{1}{\nu(n-1)}+\frac{1+\alpha(n-1)}{(\alpha-\nu)(n-1)}}
$$

providing the optimal selection of $q>1$ ensuring the fastest convergence of (3), (6), (7).

Example 1. Let us consider the case $n=2$ with

$$
k(x)=-\left|x_{1}\right|^{1+2 \nu} \operatorname{sign}\left(x_{1}\right)-\left|x_{2}\right|^{\frac{1+2 \nu}{1+\nu}} \operatorname{sign}\left(x_{2}\right),
$$

from [3], which satisfies all required conditions. Select $\nu=-0.25, q=2$ and $\alpha=-0.125$, then all conditions of Theorem 2 are satisfied and the closed loop hybrid system (3), (6), (7) is fixed-time stable. An example of the system trajectories is given in Fig. 1.

\section{B. Fixed-time stabilization for $\nu \leq 0$}

The algorithm for generation of instants $t_{i}$ will be selected as previously in (6), and

$$
\mu_{i}=\left\{\begin{array}{ll}
q^{i \alpha}\|z(0)\|_{r}^{\theta} & \text { if } i<i^{*} \\
1 & \text { otherwise }
\end{array},\right.
$$

where $\alpha \in \mathbb{R}$ and $\theta \in \mathbb{R}$ are tuning parameters. Again, it is necessary to show that the solutions of (4), (5) are well defined for the supervisory algorithm (6), (8), and that the closed loop system converges to the origin in a fixed time.

For all $t \geq t_{i^{*}}$ the system (4), (5), (6), (8) is reduced to (2), which is finite-time or exponentially stable, and $\left\|x\left(t_{i^{*}}\right)\right\|_{r} \leq$ 1. The solutions of this system are well defined for all $t \geq$

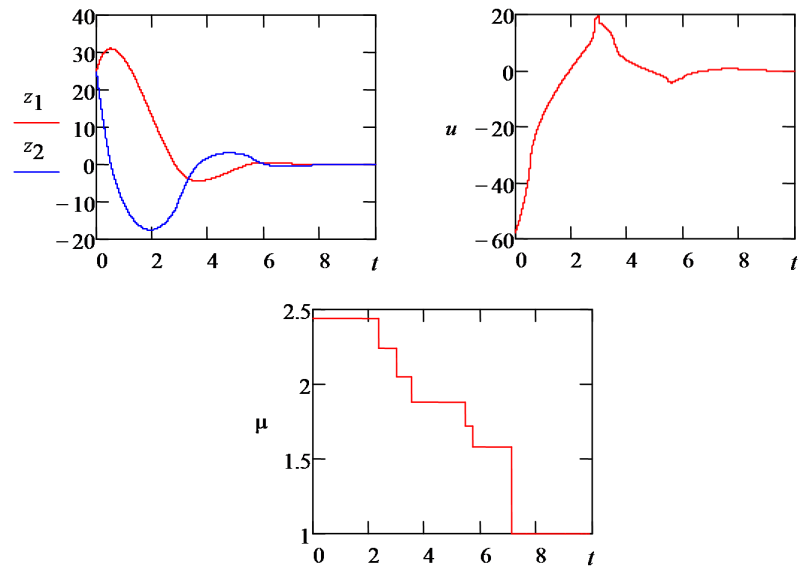

Figure 1. Fixed-time stabilization for $\nu<0$ with decreasing $\mu_{i}$

$t_{i^{*}}$, and it is necessary to consider the system behavior for $t \in\left[0, t_{i^{*}}\right)$ and estimate the time of convergence to the unit ball $t_{i^{*}}$. From (8) we obtain for all $i \in\left[0, i^{*}-1\right]$ :

$$
\begin{gathered}
M_{i+1} M_{i}^{-1}=\operatorname{diag}\left\{\left(\frac{\mu_{i+1}}{\mu_{i}}\right)^{1-k}\right\}_{k=1}^{n} \\
=\operatorname{diag}\left\{q^{\alpha(1-k)}\right\}_{k=1}^{n},
\end{gathered}
$$

and $M_{i+1} M_{i}^{-1}$ is stretching only if $\alpha<0$ (since $q>1$ ):

$$
\begin{aligned}
\left\|x\left(t_{i+1}^{+}\right)\right\|_{r} & \leq q^{\gamma(\alpha)}\left\|x\left(t_{i+1}\right)\right\|_{r} \\
& \leq q^{\gamma(\alpha)-1-i}\|z(0)\|_{r}
\end{aligned}
$$

with

$$
\gamma(\alpha)= \begin{cases}\frac{-\alpha(n-1)}{1+(n-1) \nu} & \text { if } \alpha<0 \\ 0 & \text { otherwise }\end{cases}
$$

The instant $t_{i+2}$ will be generated by (6) when $\left\|x\left(t_{i+2}\right)\right\|_{r}=q^{-2-i}\|z(0)\|_{r}$ and on the interval of time $\left[t_{i+1}, t_{i+2}\right)$ the dynamics of $x(t)$ is governed by (4) for $\mu_{i+1}=q^{(i+1) \alpha}\|z(0)\|_{r}^{\theta}$. On this interval $\left[t_{i+1}, t_{i+2}\right)$ the state $x(t)$ has to decrease in

$$
\rho=q^{1+\gamma(\alpha)}
$$

times. Let $T_{\rho}: \mathbb{R}^{n} \rightarrow \mathbb{R}_{+}$be the function of convergence in $\rho>1$ times for (2), then

$$
\begin{gathered}
t_{i+2}-t_{i+1}=\mu_{i+1}^{-1} T_{\rho}\left(x\left(t_{i+1}\right)\right) \\
\leq q^{(i+1)(\nu-\alpha)-\nu \gamma(\alpha)}\|z(0)\|_{r}^{-\theta-\nu} T_{\rho}(\xi)
\end{gathered}
$$

where $z(0)=\Lambda_{r}(\lambda) \xi$ for some $\xi$ from the unit sphere $S_{r}=$ $\left\{\xi \in \mathbb{R}^{n}:\|\xi\|_{r}=1\right\}$ as before. From the expressions above, the interval length $t_{i+2}-t_{i+1}$ is decreasing with $i$ uniformly in $\|z(0)\|_{r}$ if

$$
\alpha>\nu,-\nu \leq \theta \text {. }
$$


Indeed, in this case $\|z(0)\|_{r}^{-\theta-\nu} \leq 1$ for all $\|z(0)\|_{r} \geq 1$. Let $\bar{T}_{\rho}=\sup _{\|\xi\|_{r}=1} T_{\rho}(\xi)$, then

$$
\begin{aligned}
t_{i^{*}} & =\sum_{i=0}^{i^{*}-1}\left(t_{i+1}-t_{i}\right) \\
& \leq \sum_{i=0}^{i^{*}-1} q^{i(\nu-\alpha)-\nu \gamma(\alpha)} \bar{T}_{\rho} \\
& =q^{-\nu \gamma(\alpha)} \bar{T}_{\rho} \sum_{i=0}^{i^{*}-1} q^{i(\nu-\alpha)} \\
& \leq \frac{q^{-\nu \gamma(\alpha)}}{1-q^{\nu-\alpha}} \bar{T}_{\rho},
\end{aligned}
$$

where the fact that $q^{\nu-\alpha}<1$ has been used on the last step. Therefore, the time of convergence to the unit ball $t_{i^{*}}$ in the system (4), (5), (6), (8) is finite and independent in initial conditions, as it was necessary to show. The following result has been proven.

Theorem 3. Let for the system (3) with $\nu \leq 0$ the supervisory algorithm be selected as in (6), (8) with $q>1, \alpha>\nu$ and $-\nu \leq \theta$, then the solutions of the closed-loop system are well defined for all $t \geq 0$ and for any $z(0) \in \mathbb{R}^{n}$ the time of convergence to the unit sphere $S_{r}$ is less than

$$
\frac{q^{-\nu \gamma(\alpha)}}{1-q^{\nu-\alpha}} \bar{T}_{\rho}
$$

where $\bar{T}_{\rho}=\sup _{\|\xi\|_{r}=1} T_{\rho}(\xi)$ for the system (2) with $\rho=$ $q^{1+\gamma(\alpha)}$.

If in the conditions of the latter theorem $\nu<0$, then the system is fixed-time stable as in the case of Theorem 2 (for $\nu=0$ the system is exponentially converging to the origin form the unit sphere $S_{r}$ ), and in general Theorem 3 is a generalization of Theorem 2. However, the supervision provided in Theorem 3 is qualitatively different from the case of Theorem 2 if $\alpha>0$, since in this case $\mu_{i}$ forms an increasing sequence for $i \in\left[0, i^{*}-1\right]$.

Example 2. Let us continue to consider the same example, but with $\nu=0$ for $q=2, \alpha=0.5$ and $\theta=0.25$. All conditions of Theorem 3 are satisfied and the closed loop hybrid system (3), (6), (8) has a finite time of convergence to the unit sphere. An example of the system trajectories is given in Fig. 2.

\section{Finite-time convergence to the origin for $\nu \geq 0$}

Let us consider the problem of finite-time convergence to the origin from the unit sphere $\left(z(0) \in S_{r}\right)$ by switching among the systems having asymptotic rate of convergence, i.e. with $\nu \geq 0$. For this purpose the following supervision algorithm will be used:

$$
\begin{aligned}
t_{i+1} & =\min _{t \geq t_{i}:\|x(t)\|_{r} \leq q^{-1-i}} t, \\
\mu_{i+1} & =q^{(i+1) \alpha},
\end{aligned}
$$

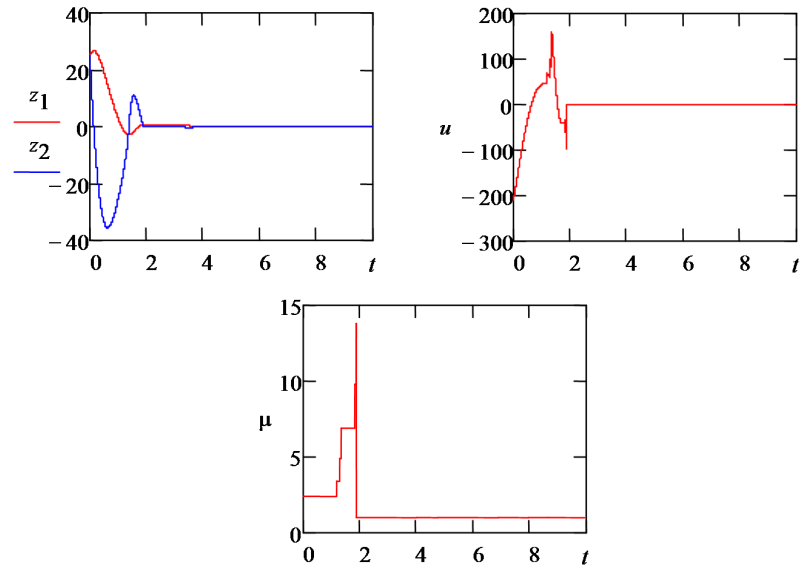

Figure 2. Finite-time convergence to $S_{r}$ for $\nu=0$ with increasing $\mu_{i}$

where $q>1$ and $\alpha>\nu$ are tuning parameters. It is necessary to show that the solutions of (4), (5) are well defined for the supervisory algorithm (9) and that the closed loop system converges to the origin in a finite-time time.

Remark 3. The difference here with respect to the cases considered previously is that there is an infinite number of switches and a Zeno behavior or a sliding mode appears. In practice the switching may be stopped after reaching a certain vicinity around the origin, i.e. when $\left\|z\left(t_{i}\right)\right\|_{r} \leq \varepsilon$ for a given $\varepsilon \in(0,1)$, which is specifying the available precision of computation, for example. In this case there is a finite number $i^{*}=\operatorname{ceil}\left[\ln _{q} \varepsilon\right]$ of switches as previously.

From (9) we obtain for all $i \geq 0$ :

$$
\begin{gathered}
M_{i+1} M_{i}^{-1}=\operatorname{diag}\left\{\left(\frac{\mu_{i+1}}{\mu_{i}}\right)^{1-k}\right\}_{k=1}^{n} \\
=\operatorname{diag}\left\{q^{\alpha(1-k)}\right\}_{k=1}^{n},
\end{gathered}
$$

then

$$
\left\|x\left(t_{i+1}^{+}\right)\right\|_{r} \leq\left\|x\left(t_{i+1}\right)\right\|_{r} \leq q^{-1-i} .
$$

Repeating the same argumentation, the instant $t_{i+2}$ will be generated by (9) when $\left\|x\left(t_{i+2}\right)\right\|_{r}=q^{-2-i}$ and on the interval of time $\left[t_{i+1}, t_{i+2}\right)$ the dynamics of $x(t)$ is governed by (4) for $\mu_{i+1}=q^{(i+1) \alpha}$. On this interval $\left[t_{i+1}, t_{i+2}\right)$ the state $x(t)$ has to decrease in $q$ times, then

$$
\begin{gathered}
t_{i+2}-t_{i+1}=\mu_{i+1}^{-1} T_{q}\left(x\left(t_{i+1}\right)\right) \\
\leq q^{(i+1)(\nu-\alpha)} T_{q}(z(0)),
\end{gathered}
$$

and the interval length $t_{i+2}-t_{i+1}$ is decreasing with $i$. Denote gain $\bar{T}_{q}=\sup _{\|\xi\|_{r}=1} T_{q}(\xi)$, then the time of convergence to 


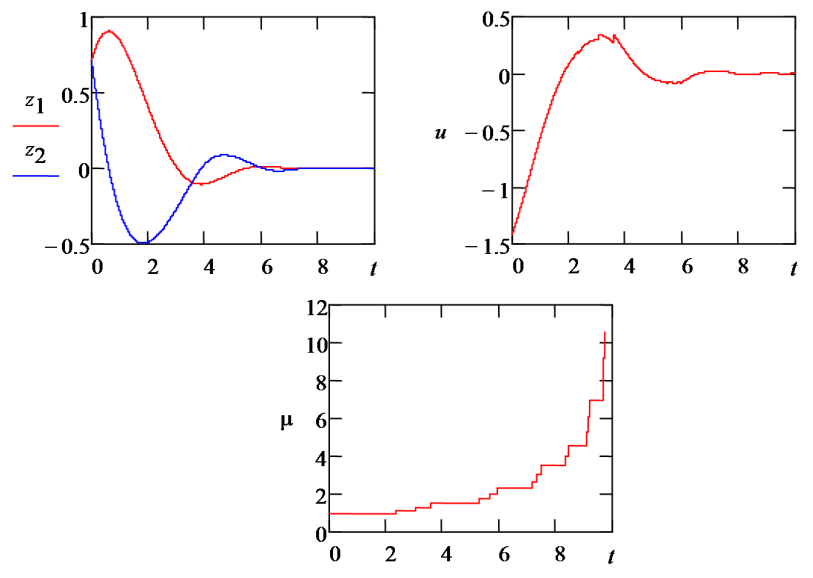

Figure 3. Finite-time convergence from $S_{r}$ to the origin for $\nu=0$ with increasing $\mu_{i}$

the origin $t_{0}$ can be estimated as follows

$$
\begin{aligned}
t_{0} & =\sum_{i \geq 0}\left(t_{i+1}-t_{i}\right) \\
& \leq \sum_{i \geq 0} q^{i(\nu-\alpha)} \bar{T}_{q} \\
& =\bar{T}_{q} \sum_{i \geq 0} q^{i(\nu-\alpha)} \\
& \leq \frac{\bar{T}_{q}}{1-q^{\nu-\alpha}}
\end{aligned}
$$

since $q^{\nu-\alpha}<1$. Therefore, the time of convergence to the origin from the unit sphere $S_{r}$ in the system (4), (5), (9) is finite and independent of initial conditions. The following result has been proven.

Theorem 4. Let for the system (3) with $\nu \geq 0$ the supervisory algorithm be selected as in (9) with $q>1$ and $\alpha>\nu$, then the solutions of the closed-loop system are well defined for all $t \geq 0$ and for any $z(0) \in S_{r}$ the time of convergence to the origin is less than

$$
\frac{\bar{T}_{q}}{1-q^{\nu-\alpha}},
$$

where $\bar{T}_{q}=\sup _{\|\xi\|_{r}=1} T_{q}(\xi)$ for the system (2).

Example 3. Continuing with the same example for $\nu=0$, $q=2$ and $\alpha=0.2$, we can observe that all conditions of Theorem 4 are satisfied and the closed loop hybrid system (3), (9) is finite-time converging to the origin from the unit sphere. An example of the system trajectories is given in Fig. 3.

\section{CONCLUSION}

The problem of convergence rate acceleration by switching among different values of parameters is addressed in this work. Different algorithms are proposed, which can be applied to homogeneous systems with all signs of degree (negative, zero, positive), and ensuring acceleration of convergence from infinity to the unit sphere or from the sphere to the origin. The obtained results are based on analysis of an auxiliary hybrid system. As a result, taking a linear feedback for linear system it is shown how to scale the gains and switch between them in order to ensure a fixed-time convergence in the closed-loop system. Different strategies are analyzed: decreasing or increasing acceleration approaching the goal set. Note that the obtained results are not related with dwell-time stability since switching stops in a finite time, with interval length between commutations converging to zero in Theorem 4 . The results are illustrated by computer simulations for a planar benchmark. Analysis of finite-time and fixed-time stability for non autonomous or hybrid systems can be directions of future research.

\section{REFERENCES}

[1] A. Bacciotti and L. Rosier. Lyapunov Functions and Stability in Control Theory. Springer, Berlin, 2nd edition, 2005.

[2] E. Bernuau, A. Polyakov, D. Efimov, and W. Perruquetti. On extension of homogeneity notion for differential inclusions. In Proceeding of the European Control Conference, pages 2204-2209, Zurich, 2013.

[3] S. P. Bhat and D. S. Bernstein. Geometric homogeneity with applications to finite-time stability. Mathematics of Control, Signals and Systems, 17:101-127, 2005.

[4] Y. Dvir and A. Levant. Accelerated twisting algorithm. Automatic Control, IEEE Transactions on, 60(10):2803-2807, Oct 2015.

[5] Y. Dvir and A. Levant. Sliding mode order and accuracy in sliding mode adaptation and convergence acceleration. In Xinghuo Yu and Mehmet Önder Efe, editors, Recent Advances in Sliding Modes: From Control to Intelligent Mechatronics, volume 24 of Studies in Systems, Decision and Control, pages 129-153. Springer International Publishing, 2015.

[6] C. Edwards and S. Spurgeon. Sliding mode control: theory and applications. Taylor and Francis, 1998.

[7] A.F. Filippov. Differential Equations with Discontinuous Righthand Sides. Kluwer Academic Publishers, 1988.

[8] H. K. Khalil. Nonlinear Systems. NJ 07458. Prentice-Hall, Upper Saddle River, 1996.

[9] A. Levant. Homogeneity approach to high-order sliding mode design. Automatica, 41(5):823-830, 2005.

[10] E. Moulay and W. Perruquetti. Finite time stability and stabilization of a class of continuous systems. Journal of Mathematical Analysis and Application, 323(2):1430-1443, 2006.

[11] E. Moulay and W. Perruquetti. Finite time stability conditions for non autonomous continuous systems. International Journal of Control, 81(5):797-803, 2008.

[12] A. Polyakov. Nonlinear feedback design for fixed-time stabilization of linear control systems. IEEE Transactions on Automatic Control, 57(8):2106-2110, 2012.

[13] L. Rosier. Homogeneous Lyapunov function for homogeneous continuous vector field. Systems \& Control Letters, 19:467-473, 1992.

[14] E. Roxin. On finite stability in control systems. Rendiconti del Circolo Matematico di Palermo, 15:273-283, 1966.

[15] V.I. Zubov. On systems of ordinary differential equations with generalized homogenous right-hand sides. Izvestia vuzov. Mathematica., 1:80-88, 1958 (in Russian). 